\title{
Self-organized Spectrum Access in Small-cell Networks with Dynamic Loads
}

\author{
Ducheng Wu, Qihui Wu, Yuhua Xu \\ PLA University of Science and Technology, China \\ [e-mails:wuducheng@foxmail.com,wuqihui2014@sina.com, yuhuaenator@gmail.com] \\ *Corresponding author: Yuhua Xu
}

Received August 18, 2015; revised December 4, 2015; accepted January 10, 2016;

published May 31, 2016

\begin{abstract}
This paper investigates the problem of co-tier interference mitigation for dynamic small- cell networks, in which the load of each small-cell varies with the number of active associated small-cell users (SUs). Due to the fact that most small-cell base stations (SBSs) are deployed in an ad-hoc manner, the problem of reducing co-tier interference caused by dynamic loads in a distributed fashion is quite challenging. First, we propose a new distributed channel allocation method for small-cells with dynamic loads and define a dynamic interference graph. Based on this approach, we formulate the problem as a dynamic interference graph game and prove that the game is a potential game and has at least one pure strategy Nash equilibrium (NE) point. Moreover, we show that the best pure strategy NE point minimizes the expectation of the aggregate dynamic co-tier interference in the small-cell network. A distributed dynamic learning algorithm is then designed to achieve NE of the game, in which each SBS is unaware of the probability distributions of its own and other SBSs' dynamic loads. Simulation results show that the proposed approach can mitigate dynamic co-tier interference effectively and significantly outperform random channel selection.
\end{abstract}

Keywords: Small-cell networks, distributed channel allocation, dynamic load, interference mitigation, potential game 


\section{Introduction}

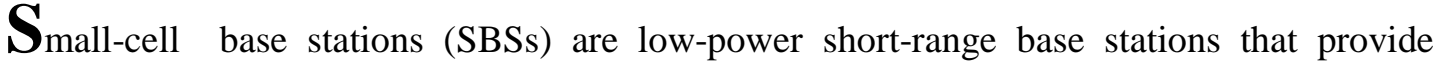
wireless services to users. Small-cell technologies can greatly improve radio resource reuse efficiency and are seen as one of the most promising solutions for the rapid growth of wireless data services and which could prove useful in satisfying the requirement of ubiquitous access [1-4]. When the number of SBSs in a given area is increased, a need arises for the mitigation of co-tier interference to exploit the benefits promised this technology [5, 6]. However, as most SBSs are deployed with minimum intervention from end users and service providers, centralized resource allocation is difficult to achieve. In this paper, we focus on self-organizing spectrum allocation for small-cell networks under the constraint of mitigating co-tier interference.

There are some existing distributed spectrum allocation solutions, using e.g., reinforcement-learning based approaches [7], hierarchical dynamical games [8] and evolutionary games [9]. However, most existing works assume that there is only one terminal user associated with each small-cell and the user remains in contact with its associated SBS all the time. In practice, in small-cell networks, more than one user can associate with each SBS and may connect or disconnect with their corresponding SBSs according to their specific service demand at non-deterministic moments in time [10]. Thus, the loads (number of active SUs) of small-cells may change dynamically and vary widely within certain (short) time periods . This paper focuses on the intractable problem of spectrum allocation for different and dynamic loads of small-cells.

In this paper, we resort to a game theory-based self-organizing approach to allocate channels among the SBSs. This approach is based on the following assumptions: first, there is no centralized controller and, second, the small-cells make decisions selfishly and autonomously. Due to the dynamics of the loads, the existing fixed- channel allocation method, in which each SU is allocated a fixed channel for each small-cell, is not applicable when the number of users is larger than the number of dedicated channels. What's more, considering the dynamics of SU loads, channels with low interference will not be used efficiently when the SUs who occupy these channels are inactive. Thus, we propose a new distributed channel allocation method with different channel priorities, which can be apllied in overload situations without regard to serving queues and buffers. In the proposed channel allocation method, channels are not allocated to specific SUs, and channels with lower interference are given a higher priority for use by active SUs. With this approach, the self-organized spectrum access with dynamic loads can be modeled as a sequential channel set selection problem. Therewith, we employ a dynamic interference graph to analyze the dynamic interference that occurs among the small-cells when the proposed channel allocation methods are applied . Subsequently, a dynamic interference graph game is formulated to mitigate the dynamic co-tier interference. In the proposed game, each player (SBS) tries to minimize the expectation of the received interference in selected channels.

Our main contributions can be summarized as follows:

- We investigate distributed channel allocation in dynamic small-cell networks, where the loads of the cells vary dynamically. We propose a new distributed channel allocation method for small-cells with dynamic loads.

- A dynamic interference graph is defined to analyze the interference caused by SUs connecting or disconnecting dynamically in each small-cell. The problem is formulated as an optimization of sequential channel sets selection under the constraint of 
minimizing the expectation of dynamic co-tier interference.

- We propose a game-based approach to allocate channels to small-cells with dynamic loads based on the defined dynamic interference graph. The utility function of each small-cell is defined as the sum of the received interference expectation in selected channels. We furthermore prove that the game is a potential game and has at least one pure strategy Nash equilibrium (NE) point. Moreover, the best pure strategy NE point minimizes the expectation of the aggregate dynamic co-tier interference in the small-cell network.

- We design a distributed dynamic learning algorithm to achieve Nash equilibria for the game, in which each SBS is not aware of the probability distributions of its own and the others' dynamic loads.

The rest of this article is organized as follows. In Section 2, we review the related work. In Section 3, we present the system model and problem formulation. In Section 4, we propose a dynamic interference graph game and analyze the existence of Nash equilibria. In Section 5, we propose a distributed dynamic learning algorithm and present simulation results. Finally, Section 6 presents our conclusions and summarizes our findings

\section{Related Work}

The problem of resource allocation and co-tier interference mitigation in small-cell networks has been investigated widely [11-14]. In many existing works [15-17], a central management system or controller is necessary for handling resource allocation and interference mitigation, which leads to significant signaling overhead between the controller and the small-cells. In [15], the authors utilized a femtocell management system to avoid co-channel and co-tier interference. In [16], the authors proposed a semi-centralized interference management scheme, which was based on joint clustering and resource allocation for small-cells. However, it is difficult to implement centralized controlling or planning to allocate resources because most small-cells are deployed in an ad-hoc manner with minimum intervention from the end users and the service providers.

Some researchers applied different game models to solve this problem in a distributed manner, e.g., coalition games [18, 19], evolutionary games [20], Bargaining games [21] and graphical games [22-25]. In [18], a coalition game model was used to enable small-cells to cooperate with each other for sharing resources and managing interference. In [22], the authors applied a graph-based model to characterize the MAC-layer interference relationship among the cells. In [23], the authors proposed two special cases of local cooperative game to study the problem of opportunistic spectrum access, in which the users need to exchange information with their neighboring users. In [24], the authors investigated the problem of channel selection for MAC-layer interference mitigation with a proposed dynamic graphic game in the dynamic and distributed environment. However, in [22-25] , they assumed that one cell can only select one channel to transmit. Moreover, the proposed MAC-layer interference is not suitable for the small-cell networks without the multiple access control mechanisms, such as CSMA and Aloha.

All the aforementioned works have paid less attention on the dynamic loads of small-cells. It should be pointed out that [10, 26, 27] have conducted some preliminary researches on load-aware spectrum access for small-cell networks. However, even in [10, 26], it is assumed that the loads of small-cells are static and unchanged. In [27], the authors resorted to a convex optimization approach to maximise the energy efficiency of dynamic wireless networks. In 
practice, in small-cell networks, the loads and channels requested of the cells change dynamically and may vary widely during a particular (short) time period. In this paper, we propose a fully distributed and self-organizing spectrum access approach with dynamic loads in small-cell networks.

\section{System Model and Problem Formulation}

\subsection{System model}

Let us consider a small-cell network (SCN), where small-cell base stations (SBSs) are independently deployed in random locations. Small-cells transmit on orthogonal channels compared to macro-cells, thus cross-tier interference is avoided in such networks. In this paper, we will only consider co-tier interference. It is assumed that there are $N$ small-cells, i.e. $\mathcal{N}=\{1,2, \ldots, N\}$, and there are $M$ dedicated channels available for each small-cell to transmit, i.e. $\mathcal{M}=\{1, \ldots, M\}$. Each cell $n$ offers traffic services to $K_{n}$ small-cell users (SUs). It is assumed that the transmission link between SBS $n$ and a SU only occupies one channel at each (short) time period. In this paper, we only consider downlink transmission.

It is assumed that each SU may be in one of two states, namely "Active" (A) and "Sleep" (S). In the "Active" state, a SU receives data from its associated small-cell, while, in the "Sleep" state, the SU has no communication demand and does not occupy any channels to communicate with the associated small-cell . The "Active-Sleep" model can be represented by a simple two-state Markov chain. Taking an arbitrary chosen SU as an example, its two-state Markov chain is shown in Fig. 1. The state transition probabilities $\left(p^{A A}, p^{A S}, p^{S A}, p^{S S}\right)$ of each SU are different and fixed. Thus, it can be concluded that the probabilities of each SU in each small-cell being active ( $\alpha$ ) or sleep ( $\beta$ ) are fixed and different, with $\alpha+\beta=1$.

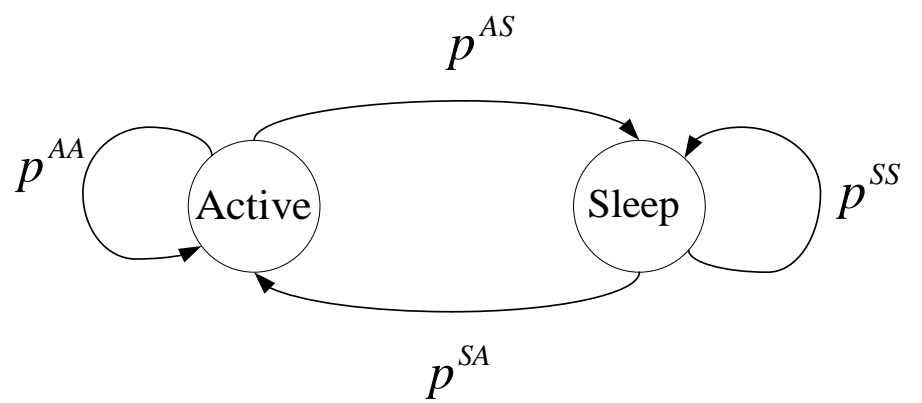

Fig. 1. Two-state Markov chain of an arbitrary chosen SU.

Because the probability of a SU being active is fixed, we can conclude that the probability of the event, in which a small-cell $n$ serves $h$ active SUs and requires $h$ channels to meet the instantaneous load, is fixed. The corresponing probability $\theta_{n h}$ can be expressed as

$$
\theta_{n h}=\sum_{\left|S \mathcal{U}_{n}^{A}\right|=h} \prod_{s u_{n i} \in \mathcal{S} \mathcal{U}_{n}^{A}} \alpha_{n i} \prod_{S u_{n j} \in \mathcal{S} \mathcal{S}_{n}^{S}} \beta_{n j}
$$

where $\mathcal{S U}_{n}^{A}$ is the active $\mathrm{SU}$ set in small-cell $n$ and $\mathcal{S U}_{n}^{S}$ is the sleeping $\mathrm{SU}$ set, and $s u_{n i}$ is 
the $i^{\text {th }}$ small-cell user associated with small-cell $n$. It is clear that $\sum_{h=0}^{K_{n}} \theta_{n h}=1$.

Considering that the load in each small-cell $n$ changes dynamically according to $\left\{\theta_{n h}\right\}$, we propose a distributed channel allocation method for small-cells with dynamic loads. In this method, each small-cell $n$ selects a sequential channel set to transmit, which can be expressed as

$$
C_{n}=\left\{c_{n 1}, c_{n 2}, \ldots, c_{n Z_{n}}\right\}
$$

where $Z_{n}=\min \left\{K_{n}, M\right\}$, and $c_{n k} \in \mathcal{M}, 0<k \leq Z_{n}$. Small-cell $n$ will prioritize these $Z_{n}$ selected dedicated channels. At any moment, each active SU can occupy an idle channel in the ordered channel set $C_{n}$ instead of occupying a particular channel in each time period.

More specifically, for channels $k$ and $k^{\prime}$ belonging to the $Z_{n}$ selected channels available for the small-cell $n$, if $k<k^{\prime}$, we say that for the cell $n, c_{n k}$ has a higher priority to be used for transmission than $c_{n k^{\prime}}$. In other words, with $i$ active SUs in small-cell $n$ at a particular time period, the first $i$ channels in $C_{n}$, i.e. the $i$ channels with the highest priority, will be picked out for transmission. Thus, to achieve good performance, channels with lower interference should be given a higher priority. The distribute channel allocation needs temporary access control provided by SBSs when the loads change.

Then, we can conclude that the probability of channel $c_{n k}$ being used to transmit by small-cell $n$ is given by:

$$
\vartheta_{n k}=\sum_{h=k}^{K_{n}} \theta_{n h}
$$

It is clear that $1 \geq \vartheta_{n 1} \geq \ldots \geq \vartheta_{n K_{n}}$. The ordering of the transmission probability of each channel is consistent with the corresponding priorities. In particular, when $c_{n k}=m(m \in \mathcal{M})$, we set $\vartheta_{n k}=\vartheta_{n}^{c_{n k}}=\vartheta_{n}^{m}$. For a small-cell $n$, the probability set of each channel $c_{n k}\left(c_{n k} \in C_{n}\right)$ being occupied for transmission is represented as

$$
\Theta_{n}=\left\{\vartheta_{n 1}, \vartheta_{n 2}, \ldots, \vartheta_{n Z_{n}}\right\}
$$

With the sequential channel set of each small-cell selected, we can examine how the interference among the small-cells varies with the dynamic loads. The statistical dynamic interference relationship can be depicted using a dynamic interference graph. Such a graph of a small-cell network with three small-cells is shown in Fig. 2. It is noted from Fig. 2 that, small-cells 1 and 3 will only interfere with each other when they both transmit information in channel 3 simultaneously. For example, the probability that small-cell 1 and 3 interfere with each other in channel 3 is $\vartheta_{1}^{3} \times \vartheta_{3}^{3}$. 


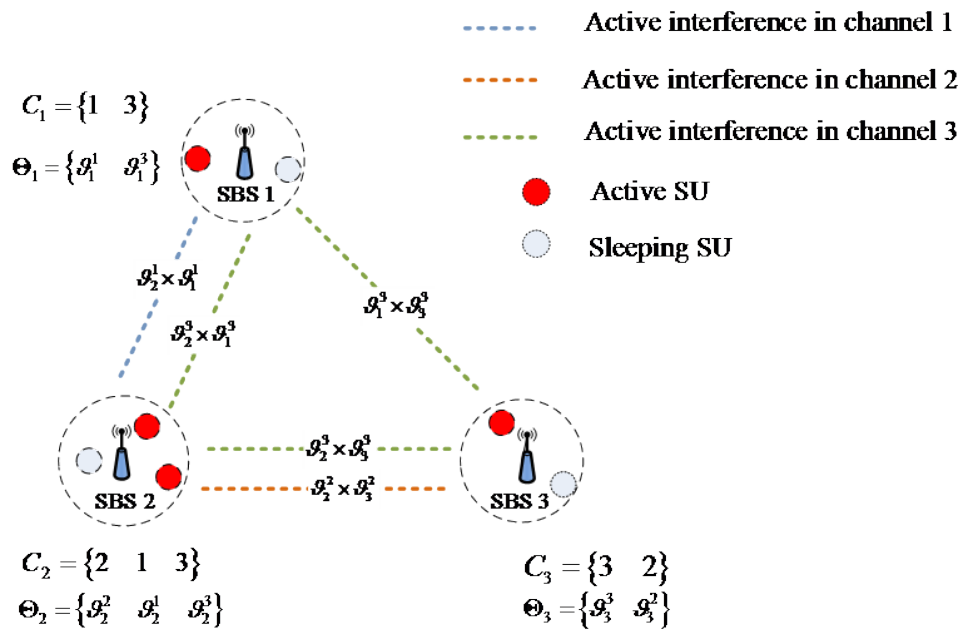

Fig. 2. An dynamic interference graph of the considered small-cell network with three small-cells.

To take a simple specific example, we assume that the distance between any two small-cells and the SBSs' transmission power in Fig. 2 are the same. Thus, when any two small-cells transmit in the same channel simultaneously, the received interference in both these small-cells is the same and can be denoted as $I$. Let us assume that the active probabilities of SUs in these 3 small-cells are $\left\{\alpha_{11}=0.6, \alpha_{12}=0.4\right\},\left\{\alpha_{21}=0.6, \alpha_{22}=0.5, \alpha_{23}=0.4\right\}$ and $\left\{\alpha_{31}=0.8, \alpha_{32}=0.5\right\}$. Then, based on (3), we have $\Theta_{1}=\{0.76,0.24\}$, $\Theta_{2}=\{0.88,0.38,0.12\}$ and $\Theta_{3}=\{0.9,0.4\}$. With the channel sets for cells 1 and 3 being $\left\{c_{11}=1, c_{12}=3\right\}$ and $\left\{c_{31}=3, c_{32}=2\right\}$ respectively, the interference probability in channel 3 between small-cell 1 and small-cell 3 is $0.24 \times 0.9=0.216$. The average interference affecting SBS 2 in channels 1,2 and 3 are then calculated as $\{0.76 I, 0.4 I, 1.14 I\}$.

Thus, the sequential channel set of small-cell 2 should be $\left\{c_{21}=2, c_{22}=1, c_{23}=3\right\}$. For small-cell 2, a diagram illustrating the dynamic load and the channel selection based on that load is shown in Fig. 3. The loads of small-cell 2 in six time periods are assumed to be $\{1,2,2,3,2,1\}$. As seen in the diagram, the utilization of channel 2 is the highest, while the utilization of channel 3 is the lowest. That is because the interference in channel 2 is the lowest, while the interference in channel 3 is the highest. Considering the dynamics of loads in small-cells, instead of allocating fixed channels to certain SUs, the proposed distributed channel allocation method with different channel priority will make most use of the channels that have low interference. 


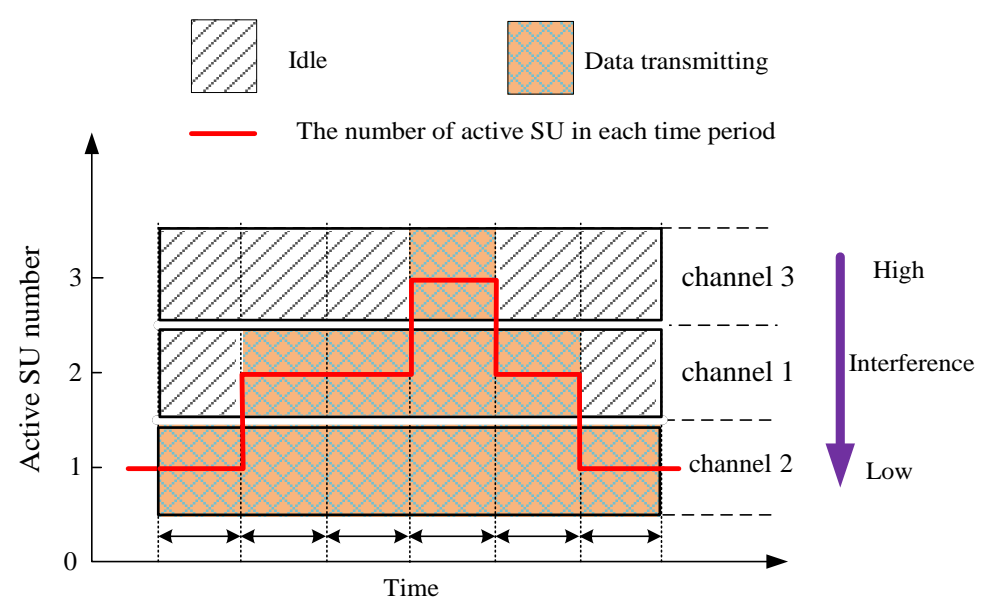

Fig. 3. An diagram illustrating dynamic load and channel selection based on that load..

According to the dynamic interference graph, the interference can be quantified in a statistical manner, and the average interference among small-cells can be calculated. This will be further elaborated in the following subsection.

\subsection{Problem formulation}

Customarily, channel allocation is performed according to the detected instantaneous channel gains of the SUs. However, due to the mobility and activity of SUs, this assumption is performed. Also, the monitoring of instantaneous channel gains of the SUs will lead to extensive signaling and huge amount of information transmission overhead. In our model, the average channel gains measured by SBSs are used for channel allocation, similar to [15]. Each small-cell performs downlink measurements of interference signal and noise power levels in each available channel. Since SUs are usually located very closely to the serving SBSs and can move around the serving SBSs, it can be assumed that the average channel gain between a SBS $n$ and a SU $s u_{n^{\prime}}$, served by another SBS $n^{\prime}$, can be approximated by the channel gain between the two SBSs ( $n$ and $n^{\prime}$ ), i.e. $\bar{g}_{s u_{n^{\prime}, n}}^{m}=g_{n^{\prime}, n}^{m}$ [15]. Following this assumption, it can be deduced that channel gains are symmetric, i.e. $\bar{g}_{s u_{n^{\prime}, n}}^{m}=g_{n^{\prime}, n}^{m}=g_{n, n^{\prime}}^{m}=\bar{g}_{s u_{n}, n^{\prime}}^{m}$, for two SBSs $n, n^{\prime}$ and two SUs $s u_{n}, s u_{n^{\prime}}$.

Let us assumed that the channel subset being occupied by SBS $n$ at time $t$ is denoted as $C_{n}(t)=\left\{c_{n 1}, C_{n 2}, \ldots, C_{n l}\right\}, C_{n}(t) \subset C_{n}$. The instantaneous received interference signal power ${ }^{1}$ by SBS $n$ in each channel $m\left(m \in C_{n}(t)\right)$ can be approximated by:

$$
I_{n m}(t)=\left\{\begin{array}{cr}
\sum_{i \in\{\mathcal{N}-n\}} \delta_{i m}(t) p_{i m} g_{i n}^{m}, & m \in C_{n}(t) \\
0, & m \notin C_{n}(t),
\end{array}\right.
$$

\footnotetext{
${ }^{1}$ The co-tier interference from some SBSs, which are not close to SBS $n$, can be catered by assuming them as a part of thermal noise as shown in [28] due to the low transmission power of small-cells. The remaining SBSs interfering with SBS $n$ which can be defined as $J_{n}=\left\{i: d_{i n}<D_{\mathrm{int}}, i \in \mathcal{N}, i \neq n\right\}$, where $d_{i n}$ is the distance between SBS $i$ and SBS $n, D_{\text {int }}$ is the defined interferce distance. Then, we can replace $i \in\{\mathcal{N}-n\}$ by $i \in J_{n}$ in (5), and the dynamic interferences can be more tractable with the following approach.
} 
where $g_{i n}^{m}$ represents the average channel gain between SBS $i$ and $n$ in channel $m, p_{i m}$ represents the transmission power of SBS $i$, and $\delta_{i m}(t)$ is the following interference indicator function:

$$
\delta_{i m}(t)= \begin{cases}1, & m \in C_{i}(t) \\ 0, & m \notin C_{i}(t) .\end{cases}
$$

Following many previous studies which focus on small-cell networks, we consider $g_{i n}^{m}$ to be mainly determined by the transmission distance, in which case $g_{i n}^{m}=g_{i n}$. To facilitate the analysis, we do not consider power control and assume that the transmission power of the SBSs in each channel is the same, i.e. $p_{i m}=p$. The proposed approach can also be applied in cases where the transmission transmit power is arbitrary and fixed.

The achieved instantaneous throughput of SBS $n$ in channel $m$ is

$$
r_{n m}=B_{0} \log _{2}\left(1+\frac{p g_{n}(t)}{I_{n m}(t)+\sigma^{2}}\right),
$$

where $g_{n}(t)$ is the instantaneous channel gain between SBS $n$ and the active SU at time $t$, $\sigma^{2}$ is the background noise, and $B_{0}$ is the bandwidth of each channel. Due to the mobility and activity of SUs, it is too hard to monitor the instantaneous channel gain $g_{n}(t)$. What's more, considering the received instantaneous interference, it is also difficult to calculate the instantaneous throughput or the average throughput. On the other hand, it is feasible to calculate the average interference of SBS $n$ in each channel over a relatively long period of time. It is clear that each SBS will prefer to give higher priority to channels with lower average interference in order to increase its servicing capacity.

Suppose that $\left(C_{n}, C_{-n}\right)$ is the sequential channel set selection profile of the small-cell network, where $C_{-n}$ is the sequential channel set selection profile of other SBSs. The average interference by SBS $n$ in channel $m$ can be described as:

$$
\bar{I}_{n m}=\lim _{T \rightarrow+\infty} \frac{1}{T} \sum_{t=1}^{T} \sum_{i \in\{\mathcal{N}-n\}} \delta_{i m}(t) p g_{i n} .
$$

It can be assumed that for each SBS $i$ with channel $m^{\prime} \in\left\{\mathcal{M}-C_{i}\right\}$, we will have $\delta_{i m^{\prime}}(t)=0, \forall t$ and $\vartheta_{i}^{m^{\prime}}=0$. Thus, (8) can be rewritten as:

$$
\bar{I}_{n m}=\sum_{i \in\{\mathcal{N}-n\}} \vartheta_{i}^{m} p g_{i n} .
$$

Because the probability that SBS $n$ occupies channel $m=c_{n k}$ to transmit its signal is $\vartheta_{n k}$ $\left(\vartheta_{n k}=\vartheta_{n}^{m}\right.$ ), the received average aggregate interference of $n$, when having a candidate channel set $C_{n}$ can be expressed as:

$$
s_{n}\left(C_{n}, C_{-n}\right)=\sum_{c_{n k} \in C_{n}} \vartheta_{n k} \bar{I}_{n c_{n k}} .
$$

The optimization goal for each small-cell is to minimize its average aggregate interference. Accordingly, a quantitative expression of the average network interference level is given by 
the following equation:

$$
U(C)=\sum_{n=1}^{N} s_{n}\left(C_{n}, C_{-n}\right)
$$

The average network interference level reflects the expectation of the aggregate dynamic co-tier interference of the network. Thus, the global objective is as follows:

$$
(\mathcal{P} 1) C_{\text {opt }}=\arg \min U(C) \text {. }
$$

This is challenging to achieve due to the dynamics of the small-cells' loads and the incomplete information constraint, i.e. i) the total number of SUs associated with each SBS is fixed, but the number of active SUs (the instantaneous load) is time-varying and the probability distribution of the dynamic load of each SBS is unknown, ii) there is no central controller and information exchange among the SBSs, which leads to each SBS not being aware of the chosen sequential channel sets and the probability distributions of loads of other SBSs. Without centralized management, we propose a distributed approach with incomplete information to solve this problem.

\section{A Dynamic Interference Graph Game Approach for Sequential Channel Set Selection}

Game theory is a potentially effective distributed approach towards handling the above problem without a central controller and complete global information. In this paper, we formulate this problem as a dynamic interference graph game, in which each player (i.e. each SBS) forms its spectrum access strategy in a distributed and autonomous manner.

\subsection{Game model}

We shall denote the dynamic interference graph game as $G_{I}=\left\{\mathcal{N},\left(\mathcal{A}_{n}\right)_{n \in \mathcal{N}},\left(u_{n}\right)_{n \in \mathcal{N}}\right\}$, where $\mathcal{N}=\{1,2, \ldots, N\}$ is the set of SBSs, $\mathcal{A}_{n}$ is the strategy space of SBS $n$, with $C_{n} \in \mathcal{A}_{n} \cdot u_{n}$ is the utility function of each player $n$, which is the inverse of the received average aggregate interference of SBS $n$, and can be expressed as:

$$
u_{n}\left(C_{n}, C_{-n}\right)=-S_{n}\left(C_{n}, C_{-n}\right) \text {. }
$$

Then the noncooperative dynamic interference graph game is expressed as follows:

$$
\left(G_{I}\right): \max _{C_{n} \in \mathcal{A}_{n}} u_{n}\left(C_{n}, C_{-n}\right) \quad \forall n \in \mathcal{N} \text {. }
$$

According to (14), it is expected that each SBS prefers to choose the channels with the lowest average interference to maximize the capacity of the small-cell. In other words, we can get $Z_{n}$ channels with the lowest average interference for each SBS $n$ by solving (14), i.e. minimizing SBS $n$ 's received average aggregate interference in all selected channels. Let us consider a scenario where the sequential channel set selection profile of other players $C_{-n}$ is fixed, in which case we have the following theorem.

Theorem 1: For each SBS $n$ withae fixed $C_{-n}$, for $C_{n}^{*}=\underset{C_{n}}{\arg \max } u_{n}\left(C_{n}, C_{-n}\right)$, where $C_{n}^{*}=\left\{c_{n 1}^{*}, c_{n 2}^{*}, \ldots, c_{n Z_{n}}^{*}\right\}$, then we have $\bar{I}_{n c_{n 1}^{*}} \leq \ldots \leq \bar{I}_{n c_{n Z_{n}}^{*}} \leq \bar{I}_{n m^{\prime}}, \forall m^{\prime} \in\left\{\mathcal{M}-C_{n}^{*}\right\}$. 
Proof: i) If $\exists h>k\left(c_{n k}^{*}, c_{n h}^{*} \in C_{n}^{*}\right)$, we have $\bar{I}_{n c_{n k}^{*}}>\bar{I}_{n c_{n h}^{*}}$. For $C_{n}^{\prime}=\left\{c_{n 1}^{\prime}, \ldots, c_{n Z_{n}}^{\prime}\right\}$, which is obtained by exchanging the positions of $c_{n k}^{*}$ and $c_{n h}^{*}$ in $C_{n}^{*}$, there have $c_{n j}^{*}=c_{n j}^{\prime}$, $\forall c_{n j}^{\prime} \in\left(C_{n}^{\prime}-\left\{c_{n k}^{*}, c_{n h}^{*}\right\}\right), c_{n k}^{*}=c_{n h}^{\prime}$ and $c_{n h}^{*}=c_{n k}^{\prime}$. Then based on (10), we obtain:

$$
\begin{aligned}
& u_{n}\left(C_{n}^{\prime}, C_{-n}\right)-u_{n}\left(C_{n}^{*}, C_{-n}\right) \\
= & \vartheta_{n k} \bar{I}_{n c_{n k}^{*}}+\vartheta_{n h} \bar{I}_{n c_{n h}^{*}}-\left(\vartheta_{n k} \bar{I}_{n c_{n k}^{\prime}}+\vartheta_{n h} \bar{I}_{n c_{n h}^{\prime}}\right) \\
= & \left(\vartheta_{n k}-\vartheta_{n h}\right)\left(\bar{I}_{n c_{n k}^{*}}-\bar{I}_{n c_{n h}^{*}}\right)>0 .
\end{aligned}
$$

However, due to $C_{n}^{*}=\underset{C_{n}}{\arg \max } u_{n}\left(C_{n}, C_{-n}\right)$, it must hold that $u_{n}\left(C_{n}^{\prime}, C_{-n}\right)<u_{n}\left(C_{n}^{*}, C_{-n}\right)$ and thus assumption i) does not hold.

ii) If $\exists m^{\prime} \in\left\{\mathcal{M}-C_{n}^{*}\right\}, c_{n k}^{*} \in C_{n}^{*}$, we have $\bar{I}_{n c_{n k}^{*}}>\bar{I}_{m^{\prime}}$. Similarly, for $C_{n}^{\prime}$ obtained by replacing $C_{n k}^{*}$ with $m^{\prime}$ in $C_{n}^{*}$, we would have $u_{n}\left(C^{\prime}, C_{-n}\right)-u_{n}\left(C_{n}^{*}, C_{-n}\right)>0$. This is contradictory with $C_{n}^{*}=\underset{C_{n}}{\arg \max } u_{n}\left(C_{n}, C_{-n}\right)$, so assumption ii) does not hold.

Therefore, through minimizing the received average aggregate interference, each SBS $n$ would get $Z_{n}$ channels with the minimum average interference. Moreover, the channel with the lowest interference would be selected with a highest priority for transmission. Theorem 1 is proved.

\subsection{Analysis of NE}

In this subsection, we examine whether Nash equilibrium (NE) of the dynamic interference graph game exist. For game $G_{I}$, an action profile $C_{N E}=\left(C_{1}^{*}, C_{2}^{*}, \ldots, C_{N}^{*}\right)$ is a pure strategy $\mathrm{NE}$ if and only if no player can improve its utility by deviating unilaterally, i.e.

$$
u_{n}\left(C_{n}^{*}, C_{-n}^{*}\right) \geq u_{n}\left(C_{n}, C_{-n}^{*}\right), \forall n \in \mathcal{N}, \forall C_{n} \in \mathcal{A}_{n}, C_{n} \neq C_{n}^{*} .
$$

Theorem 2: $G_{I}$ is an exact potential game which has at least one pure strategy NE. The optimal solution of the network average interference minimization problem is a pure strategy NE of $G_{I}$.

Proof: We construct the potential function as

$$
\begin{array}{r}
\Phi\left(C_{n}, C_{-n}\right)=-\frac{1}{2} U\left(C_{n}, C_{-n}\right) \\
=-\frac{1}{2} \sum_{n=1}^{N} u_{n}\left(C_{n}, C_{-n}\right) \\
=\frac{1}{2} \sum_{n=1}^{N} s_{n}\left(C_{n}, C_{-n}\right) .
\end{array}
$$

We see that the potential function is equal to half of the average network interference.

Suppose that an arbitrary player $n$ unilaterally changes its channel selection from $C_{n}=\left\{c_{n 1}, c_{n 2}, \ldots, c_{n Z_{n}}\right\}$ to $C_{n}^{\prime}=\left\{c_{n 1}^{\prime}, c_{n 2}^{\prime}, \ldots, C_{n Z_{n}}^{\prime}\right\}$. 
Because $\left.\vartheta_{n}^{m}\right|_{\left.m \in\left\{\mathcal{M}-C_{n}\right\}\right)}=0$, based on (9)-(13), $u_{n}\left(C_{n}, C_{-n}\right)$ can be rewritten as

$$
\begin{aligned}
u_{n}\left(C_{n}, C_{-n}\right) & =-\sum_{c_{n k} \in C_{n}} \vartheta_{n}^{c_{n k}} \bar{I}_{n c_{n k}}=-\sum_{m \in \mathcal{M}} \vartheta_{n}^{m} \bar{I}_{n m} \\
& ==-\sum_{m \in \mathcal{M}} \sum_{i \in\{\mathcal{N}-n\}} \vartheta_{n}^{m} \vartheta_{i}^{m} p g_{i n},
\end{aligned}
$$

Then the change in individual utility function caused by this unilateral change is given by

$$
\begin{aligned}
& u_{n}\left(C_{n^{\prime}}, C_{-n}\right)-u_{n}\left(C_{n}, C_{-n}\right) \\
= & -\sum_{m \in \mathcal{M}} \sum_{i \in\{\mathcal{N}-n\}} \vartheta_{n}^{\prime m} \vartheta_{i}^{m} p g_{i n}+\sum_{m \in \mathcal{M}} \sum_{i \in\{\mathcal{N}-n\}} \vartheta_{n}^{m} \vartheta_{i}^{m} p g_{i n} \\
= & \sum_{m \in \mathcal{M}} \sum_{i \in\{\mathcal{N}-n\}}\left(\vartheta_{n}^{m}-\vartheta_{n}^{\prime m}\right) \vartheta_{i}^{m} p g_{i n},
\end{aligned}
$$

where $\vartheta_{n}^{\prime m}$ is the special probability of SBS $n$ transmitting in channel $m$ out of a sequential channel candidate set $C_{n}^{\prime}$.

Based on (17) the change in the potential function caused by this unilateral change is given by

$$
\begin{aligned}
& \Phi\left(C_{n}^{\prime}, C_{-n}\right)-\Phi\left(C_{n}, C_{-n}\right) \\
= & \frac{1}{2}\left\{u_{n}\left(C_{n}^{\prime}, C_{-n}\right)-u_{n}\left(C_{n}, C_{-n}\right)\right. \\
= & \left.+\sum_{i \in\{\mathcal{N}-n\}}\left(u_{i}\left(C_{i}, C_{-i}^{\prime}\right)-u_{i}\left(C_{i}, C_{-i}\right)\right)\right\},
\end{aligned}
$$

where $u_{i}\left(C_{i}, C_{-i}^{\prime}\right)$ denotes the utility of player $i$ after the unilateral change of selection by player $n$.

Using (13) and (18), we obtain the following equation:

$$
\begin{aligned}
& u_{i}\left(C_{i}, C_{-i}^{\prime}\right)-u_{i}\left(C_{i}, C_{-i}\right) \\
= & s_{i}\left(C_{i}, C_{-i}\right)-s_{i}\left(C_{i}, C_{-i}^{\prime}\right) \\
= & \sum_{m \in \mathcal{M}} \sum_{j \in \mathcal{M}-\{i, n\}} \vartheta_{i}^{m} \vartheta_{j}^{m} p g_{i j}+\sum_{m \in \mathcal{M}} \vartheta_{i}^{m} \vartheta_{n}^{m} p g_{n i} \\
& -\left(\sum_{m \in \mathcal{M}} \sum_{j \in \mathcal{M}-\{i, n\}} \vartheta_{i}^{m} \vartheta_{j}^{m} p g_{i j}+\sum_{m \in \mathcal{M}} \vartheta_{i}^{m} \vartheta_{n}^{\prime m} p g_{n i}\right) \\
= & \sum_{m \in \mathcal{M}} \vartheta_{i}^{m}\left(\vartheta_{n}^{m}-\vartheta_{n}^{\prime m}\right) p g_{n i} .
\end{aligned}
$$

Then from (19)-(21), it can be concluded that

$$
\begin{aligned}
& \Phi\left(C_{n}^{\prime}, C_{-n}\right)-\Phi\left(C_{n}, C_{-n}\right) \\
= & \frac{1}{2}\left\{u_{n}\left(C_{n}^{\prime}, C_{-n}\right)-u_{n}\left(C_{n}, C_{-n}\right)\right. \\
& \left.+\sum_{i \in\{\mathcal{N}-n\}} \sum_{m \in \mathcal{M}} \vartheta_{i}^{m}\left(\vartheta_{n}^{m}-\vartheta_{n}^{\prime m}\right) p g_{n i}\right\} \\
= & u_{n}\left(C_{n}^{\prime}, C_{-n}\right)-u_{n}\left(C_{n}, C_{-n}\right) .
\end{aligned}
$$

Thus, it is shown that $G_{I}$ is an exact potential game in accordance with the definition of exact potential games given in [29]. The most two important properties of potential games are 
the following: i) A potential game has at least one pure strategy NE. ii) Local or global maxima of the potential function constitutes a pure strategy NE.

Based on the above properties, Theorem 2 is proved.

\section{Algorithm and Simulation}

Up to this point, we have explored the equilibrium behavior within the theoretic formulation of the dynamic interference graph game for the distributed sequential channel set selection problem. The question that remains is how the autonomous small-cells can reach equilibrium in a distributed fashion.

It is well known that some learning algorithms, for example the best response, fictitious play, spatial adaptive and log-linear learning algorithms, can converge towards pure strategy NE points for an exact potential game.

However, these algorithms are designed for static game models, in which the utilities are static and can be calculated with information exchange among players, and therefore cannot be applied in the dynamic interference graph game.

\subsection{Algorithm description}

A distributed dynamic learning algorithm, motivated by the imitative Boltzman-Gibbs algorithm with multiplicative weights [30,31], is proposed to achieve the Nash equilibria of the dynamic interference graph game.

In the proposed algorithm, without information exchange, the utility of each player is considered to be the temporal aggregate interference varying with the dynamic load of each small-cell in each iteration. Moreover, in the proposed algorithm, the SBSs do not need to know the probability distribution of their dynamic load. Algorithm 1 elaborates on this approach.

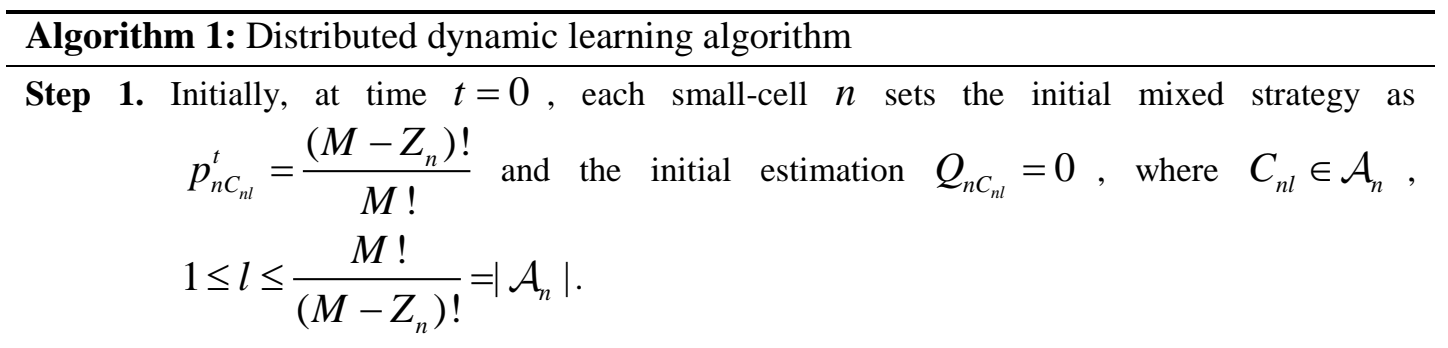

Step 2. At time $t \geq 1$, each small-cell $n$ stochastically selects an action $C_{n}^{t}$ according to its current sequential channel set selection probability vector $p_{n}^{t}$.

Step 3. All small-cells sense the interference of channels in $C_{n}^{t}$ and transmit signal based on the current load. Then, using (5) and (13), they calculate the temporal aggregate interference, which serve as the temporal utilities, i.e.

$$
u_{n}^{t}=-\sum_{c_{n k}^{t} \in C_{n}^{t}} \delta_{n c_{n k}^{t}}(t) I_{n c_{n k}^{t}}(t)
$$

In order to facilitate the implementation of the algorithm, we add a constant to the utility of each small-cell to make the utility greater than zero, i.e.

$$
r_{n}^{t}=Z_{n} I_{\text {max }}+u_{n}^{t},
$$

where $I_{\max }$ has a fixed value andis greater than or equal to the upper bound of interference 
detected in each channel.

Step 4. All the players update their estimation according to the following rules:

$$
\begin{aligned}
& Q_{n C_{n l}}^{t+1}=Q_{n C_{n l}}^{t}+\lambda_{t}\left(\frac{r_{n}^{t}}{Z_{n} I_{\max }}-Q_{n C_{n l}}\right), C_{n l}=C_{n}^{t} \\
& Q_{n C_{n l}}^{t+1}=Q_{n C_{n l}}^{t}, \quad C_{n l} \neq C_{n}^{t},
\end{aligned}
$$

where $\lambda_{t}$ is the the step factor. Finally, all the players update their mixed strategies according to the following rule:

$$
p_{n C_{n l}}^{t+1}=\frac{p_{n C_{n l}}^{t}(1+b)^{Q_{n C_{n l}}^{t}}}{\sum_{C_{n k} \in \mathcal{A}_{n}} p_{n C_{n k}}^{t}(1+b)^{Q_{n C_{n k}}^{t}}},
$$

where $b$ is the learning parameter.

Step 5. Return to Step 2 and repeat unless the stopping criterion has been met.

Because the value of $Z_{n} I_{\text {max }}$ is fixed, $\max _{C_{n} \in \mathcal{A}_{n}} r_{n}\left(C_{n}, C_{-n}\right)$ is equivalent to $\max _{C_{n} \in \mathcal{A}_{n}} u_{n}\left(C_{n}, C_{-n}\right)$. Moreover, even if we were to replace the utility $u_{n}$ by $r_{n}$, the properties of the game would not change.

Theorem 3: With sufficiently small values for $\lambda$ and $b$, the distributed dynamic learning algorithm converges to the Nash equilibria of the potential game $G_{I}$.

Proof: The detailed proof lines can be found in [28, 29].

Based on Theorems 2 and 3, we see that the distributed dynamic learning algorithm converges to the Nash equilibria of the proposed dynamic interference graph game $G_{I}$. Moreover, as discussed before, the potential function of the proposed game is equal to half of the average aggregate network interference. Thus, the proposed algorithm would achieve the desirable performance when convergence to Nash equilibria has been achieved.

\subsection{Simulation results and discussion}

In this section, we present simulations conducted for the evaluation of evaluate the performance of the proposed learning algorithm in a dynamic and distributed environment. The simulation settings were set as follows. The transmission power of each SBS in each channel was $40 \mathrm{~mW}$, the background noise was $-174 \mathrm{dBm} / \mathrm{Hz}$. The channel bandwidth was $B_{0}=500 \mathrm{kHz}$. The coverage radius of a small-cell was $10 \mathrm{~m}$. Like many previous studies [15], in order to simplify our analysis of the small-cell network, when modelling the propagation environment we only considered path loss. The path loss between two SBSs, was assumed equal to $P L=38.46+20 \log \left(d_{i j}\right)$, where $d_{i j}$ is the distance between SBSs $i$ and $j$.

1) Convergence behavior: We analyzed the convergence behavior of distributed channel allocation with the proposed dynamic learning algorithm. We considered a small-cell network with 20 small-cells in a square region of $100 \mathrm{~m} \times 100 \mathrm{~m}$. All the small-cells were randomly located. The number of dedicated channels is $M=3$ and there were 1-3 SUs for each small-cell, i.e. the number of the associated SUs $\left(K_{n}\right)$ of each small-cell $n$ was randomly selected from 1-3. The active probability of each SU was randomly selected from $[0,1]$ and 
then the probability distribution of the dynamic load of each SU was determined. We set $b=0.2$, and $\lambda=0.1$.

The evolution of action probabilities of an arbitrary chosen small-cell are shown in Fig. 4. In this simulation, the number of SUs in the chosen small-cell is 2. Each selected sequential channel sets of this cell contains 2 of the 3 dedicated channels. Thus, the number of the available actions is 6 . It noted from the figure that the cell converges to a pure strategy in about 80 iterations, and in this case stable sequential channel set is $\{2,3\}$.

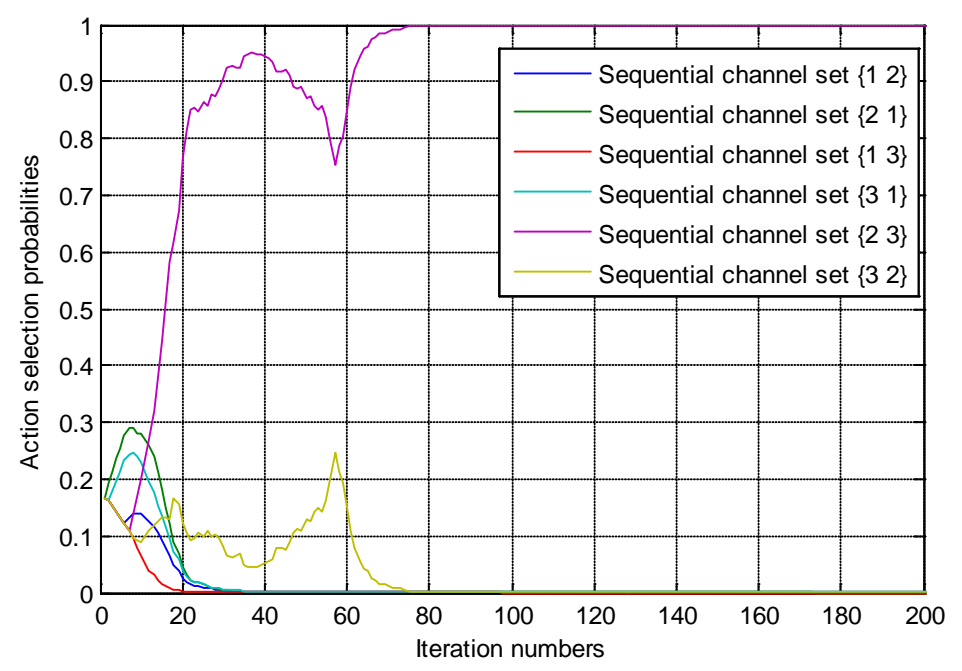

Fig. 4. Evolution of action probabilities of an arbitrary chosen small-cell.

It is clear that each small-cell must select at least one channel to serve the SUs, which will be the channel with the highest transmission priority in the sequential channel set. The evolution of the number of users for which a particular channel is selected as the highest transmission priority channel is shown in Fig. 5. It is noted that the network converges in about 200 iterations.

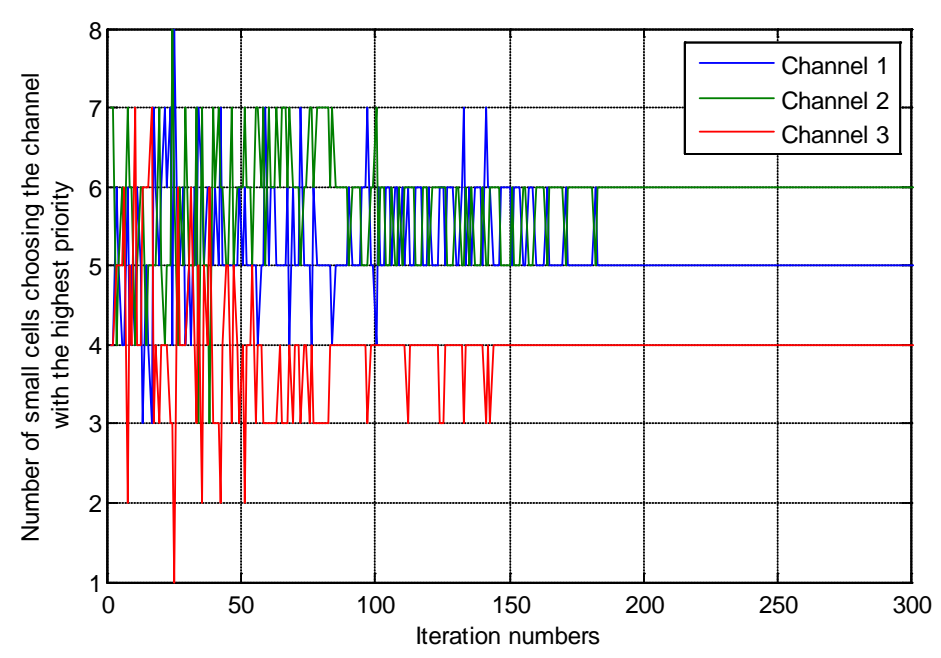

Fig. 5. Number of small-cells choosing the channel with the highest priority. 
2) Performance evaluation: First, we evaluate the interference performance assuming an identical "Active" probability $\alpha$ for all SUs. We varied $\alpha$ from 0.2 to 0.6 and set $M=4$, $K_{n}=3$. Specifically, we assumed that all small-cells were aware of all the necessary information of active probabilities and channel sets selection. The utilities of the small-cells were calculated using (18). Thus, the NE of the proposed game can be accurately achieved. The results were obtained by simulating 1000 independent trials. The interference performance for a the random channel allocation process, the best and worst NE of the proposed game are shown in Fig. 6. As shown in the figure, the NE solutions give rise to much less network interference than random allocation does.

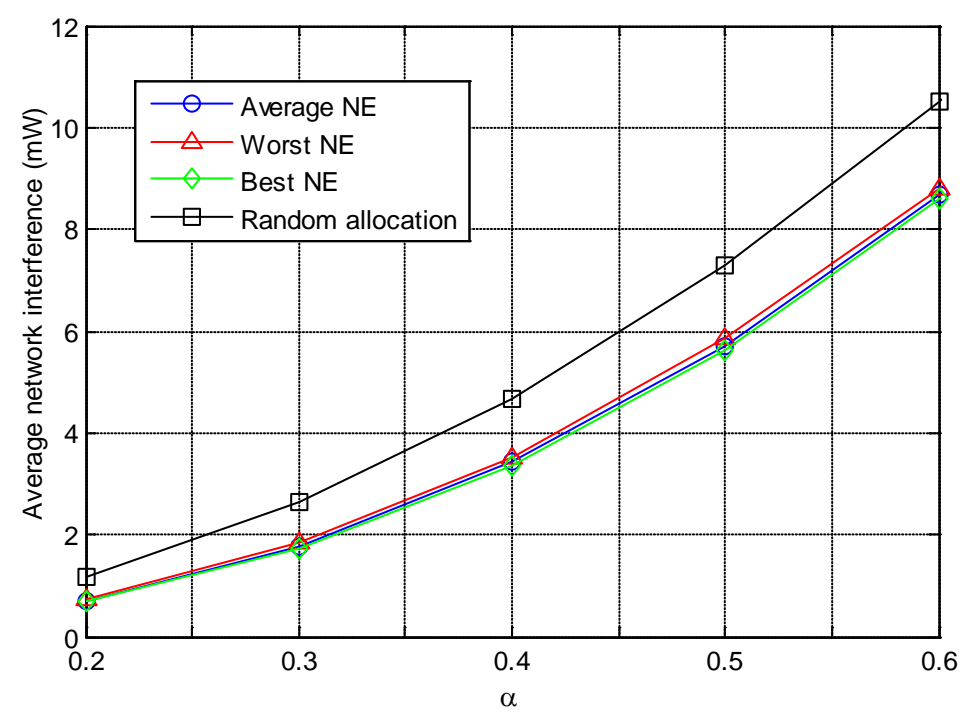

Fig. 6. Average network interference levels with varying identical $\alpha$ from 0.2 to 0.6 and $M=4$,

$$
K_{n}=3 \text {. }
$$

Second, in order to further evaluate the interference performance and the fairness performance, we consider a special scenario where $M=4, K_{n}=3$, and $\alpha_{n 1}=0.6$, $\alpha_{n 2}=0.4, \alpha_{n 3}=0.2, n \in \mathcal{N}$. In the following section, we vary the the number of small-cells from 15 to 25 , and present the performance evaluation results. In this scenario, the probability distributions of the cells' dynamic loads are the same. If the probability distributions are different, the demanded channels by each small-cell are different, and fairness is hard to calculate under these conditions. The utilities of small-cells were calculated using (18). The interference performance for random allocation, the best and worst NE of the proposed game are shown in Fig. 7. 


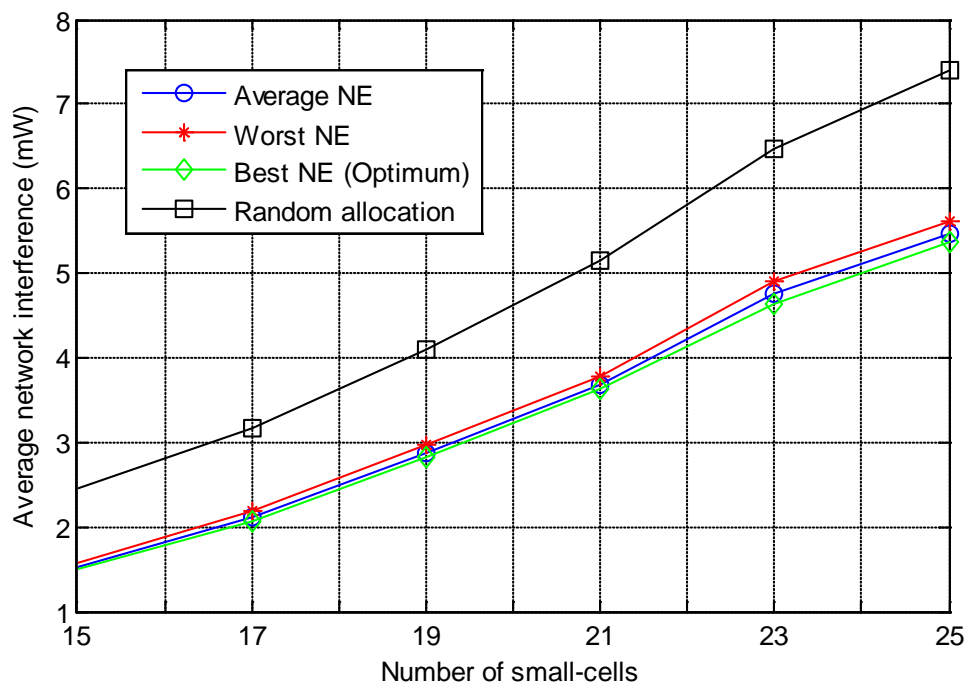

Fig. 7. Average network interference levels with $M=4, K_{n}=3$, and $\alpha_{n 1}=0.6, \alpha_{n 2}=0.4$,

$$
\alpha_{n 3}=0.2, n \in \mathcal{N} \text {. }
$$

As shown in the figure, random allocation achieves the worst performance and the NE solutions fare much better. In addition, we can see that the performance gap between the best $\mathrm{NE}$ and the worst NE is very small, i.e. the performance of the NE solution is very stable.

We then proceed to calculate Jain's fairness index (JFI) [32] of the small-cell networks with each SBS's received aggregate interference ( $s_{n}$ in (10)). The result is shown in Fig. 8. It is noted from the figure that the JFI value achieved by the NE is significantly higher than that of random allocation.

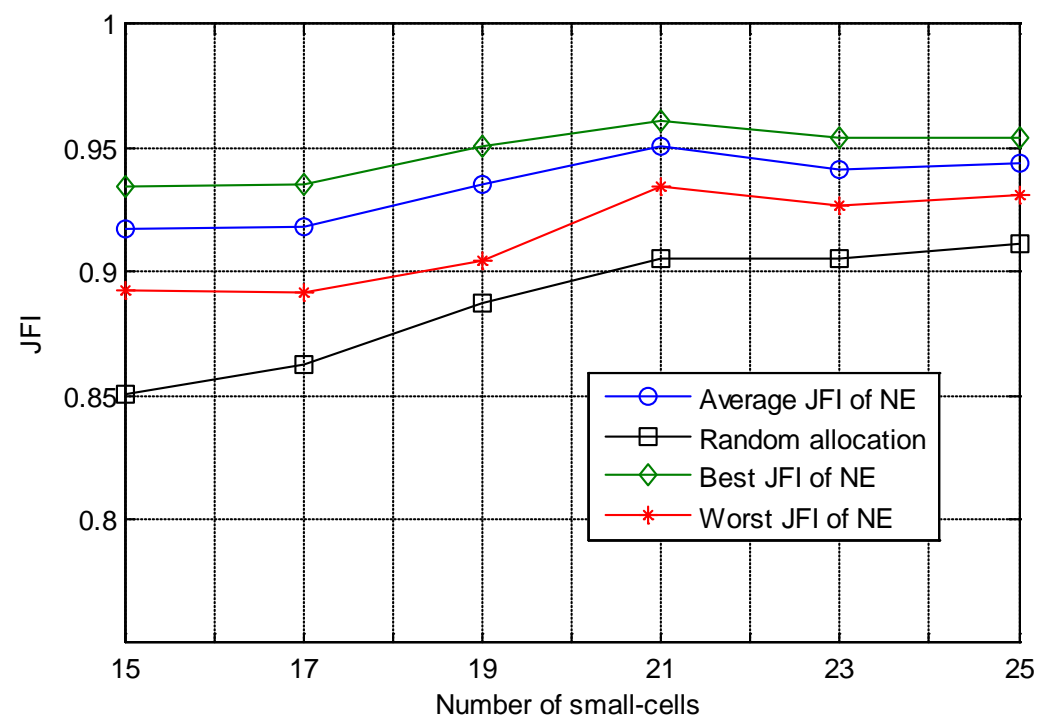

Fig. 8. JFI of small-cell networks with $M=4, K_{n}=3$, and $\alpha_{n 1}=0.6, \alpha_{n 2}=0.4, \alpha_{n 3}=0.2$, $n \in \mathcal{N}$. 
Third, we evaluated interference and throughput performance in a more common scenario. We set $M=4, K_{n}$ was randomly selected from $\{1,2,3\}$ and the active probabilities of SUs were randomly selected from $[0,1]$ in each independent trial. Each SBS did not know the probability distributions of its own and other SBSs' dynamic loads. The utilities of small-cells were calculated using (23). We simulated 1000 independent trials and then calculated the average results. The average network interference levels when varying the number of small-cells are shown in Fig. 9. It can be noted from the figure that the proposed distributed channel allocation significantly outperforms the random allocation. As the number of small-cells in a given region increases, the average interference level increases.

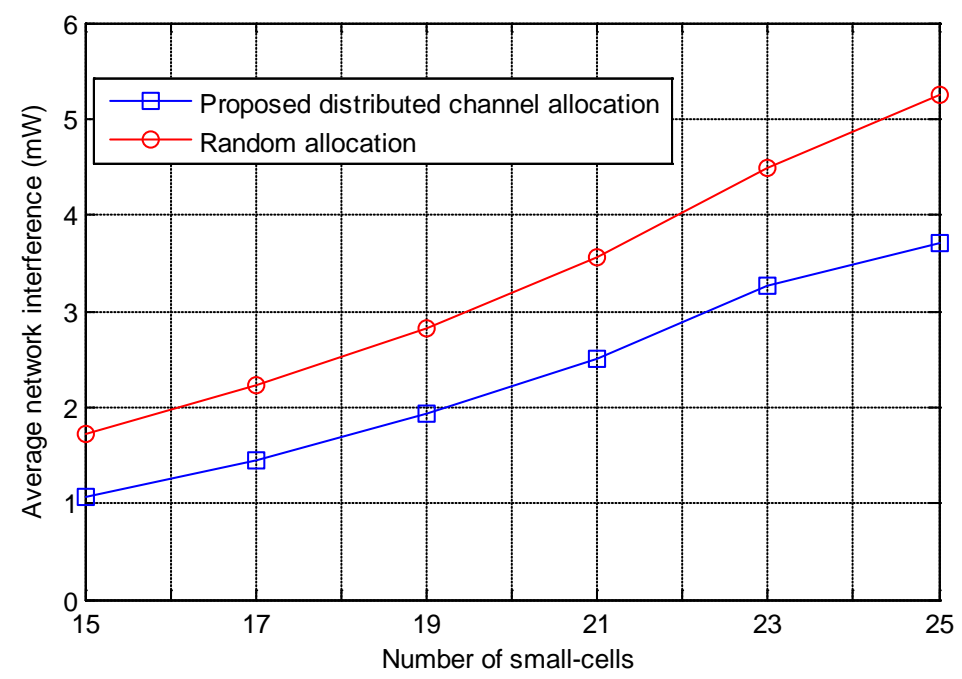

Fig. 9. The average aggregate interference levels with $M=4$, randomly selected $K_{n}$ and active probabilities $\left(1 \leq K_{n} \leq 3,0 \leq \alpha \leq 1\right)$.

The average achieved throughput of the network when varying the number of small-cells is shown in Fig. 10. In practice, due to restrictions on the capabilities (coding and modulation) of devices, the signal to interference plus noise ratio (SINR) must be greater than a predetermined threshold. We set the SINR threshold as $5 \mathrm{~dB}$ and the transmission rate of each available channel at $1 \mathrm{Mbps}$. For a given channel, a cell was allowed to transmit data with a rate of $1 \mathrm{Mbps}$ only when the temporal SINR is greater than $5 \mathrm{~dB}$. It can be seen from the figure that the average throughput, which is achieved using the distributed channel allocation method, is much higher than the random allocation strategy. As the number of small-cells increases, the average achieved throughput decreases. 


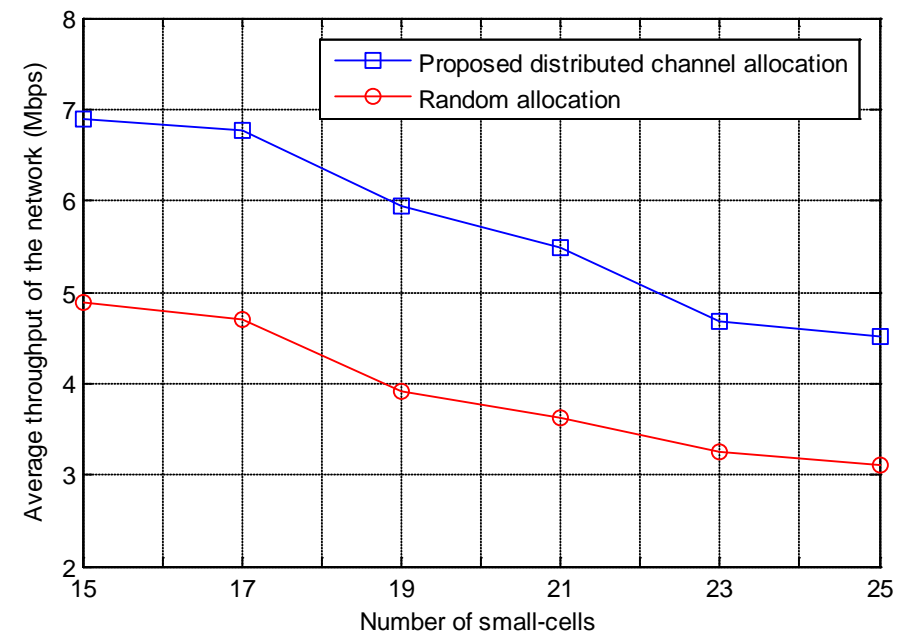

Fig. 10. The average achieved throughput of networks with $M=4$, randomly selected $K_{n}$ and active probabilities $\left(1 \leq K_{n} \leq 3,0 \leq \alpha \leq 1\right)$.

Lastly, we consider three small-cell networks with i) $M=3,1 \leq K_{n} \leq 3$, ii) $M=4$, $1 \leq K_{n} \leq 3$, and iii) $M=3,1 \leq K_{n} \leq 4$, respectively. The comparison results obtained by distributed channel allocation with different dedicated channels and different SU numbers are shown in Fig. 11. It is noted from the figure that as the number of dedicated channels increases, the average interference decreases accordingly. It also noted from the figure that the number of SUs in each small-cell increases, the average interference increases. The average achieved throughput of these networks is shown in Fig. 12. It can be seen from Fig. 11 and Fig. 12 that the more abundant channel resources are, the lower network interference will be present and the higher the achieved throughput will be.

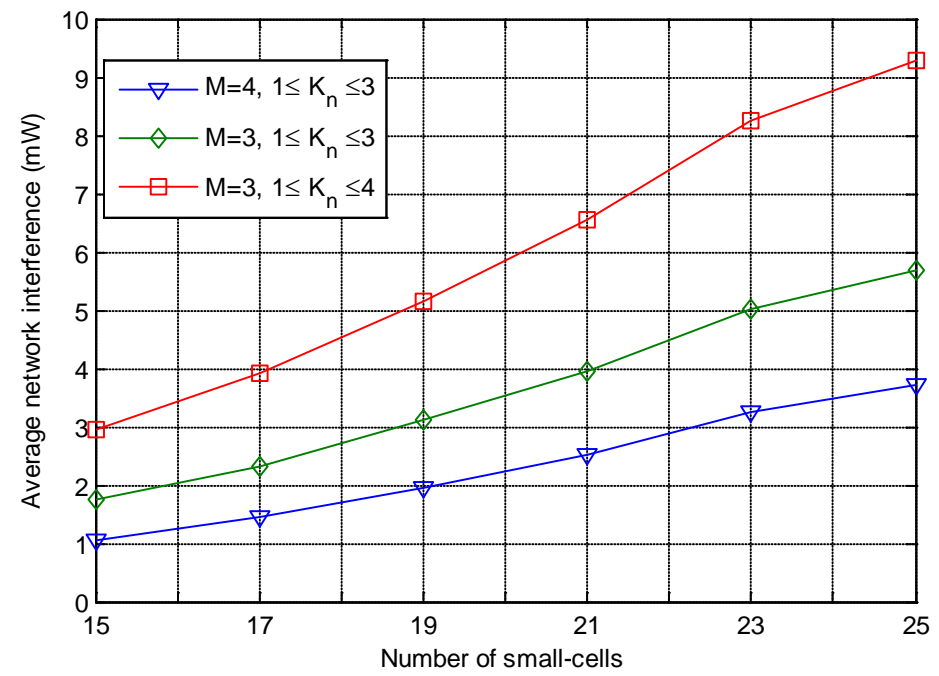

Fig. 11. Average network interference levels of small-cell networks with different numbers of dedicated channels and SUs per cell. 


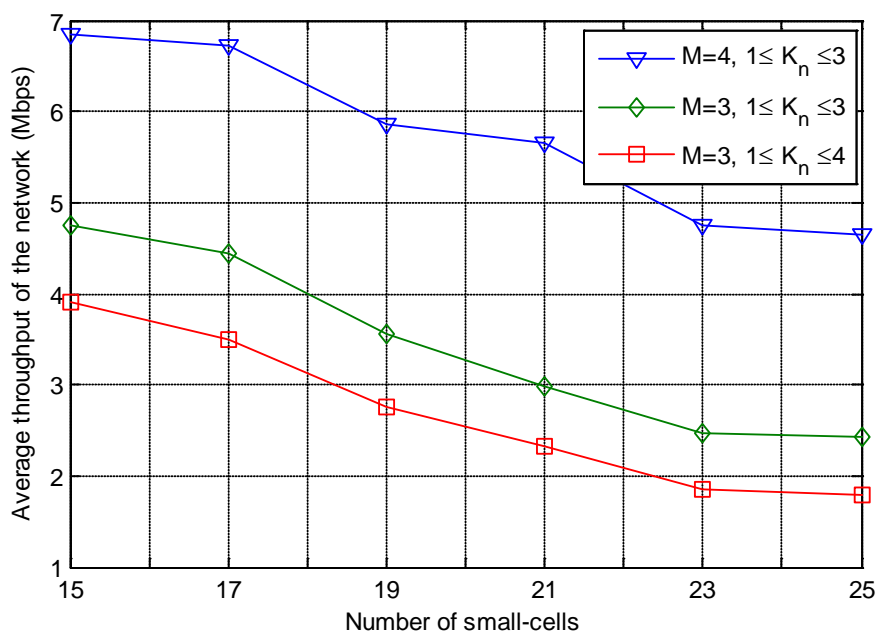

Fig. 12. The average achieved throughput of networks with different numbers of dedicated channels and SUs per cell.

\section{Conclusion}

In this paper, we focused on the mitigation of co-tier interference and studied the issue of distributed channel allocation in small-cell networks with dynamic loads. We proposed an allocation method for small-cells with dynamic loads. In particular, the distributed channel allocation method pertains to cases when the load was larger than the number of dedicated channels. In the absence of a central controller and information exchange between the cells, we defined a dynamic interference graph and formulated the problem as a dynamic interference graph game. We proved that the game is a potential game and has at least one pure strategy NE point. A distributed dynamic learning algorithm was then designed to achieve the NE of the game, in which each SBS doesn't know the probability distributions of its own and the others' dynamic loads. Simulation results showed that the proposed approach could mitigate dynamic co-tier interference effectively and outperform random selection significantly. In our future work, we aim to consider the distributed power control and channel allocation problem synthetically to meet the required transmission rates of users and reduce the aggregate power consumption.

\section{References}

[1] V. Chandrasekhar, J. Andrews, and A. Gatherer, "Femtocell networks: a survey," IEEE Commun. Mag., vol. 46, no. 9, pp. 59-67, Sep. 2008. Article (CrossRef Link)

[2] Y. Sun, R. CJover, and X. Wang, "Uplink interference mitigation for OFDMA femtocell networks,” IEEE Trans. Wireless Commun., vol. 11, no. 2, pp. 614-625, Feb. 2012. Article (CrossRef Link)

[3] C. X. Wang, F. Haider, X. Gao, et al.,"Cellular architecture and key technologies for 5G wireless communication networks,” IEEE Commun. Mag., vol. 52, no. 2, pp. 122-130, Feb. 2014. Article (CrossRef Link)

[4] M. Jo, T. Maksymyuk, R. L. Batista, et al, "A Survey of Converging Solutions for Heterogeneous Mobile Networks, ” IEEE Wireless Communications, vol. 21, no. 8, pp.54-62, Dec, 2014. Article (CrossRef Link) 
[5] V. Chandrasekhar and J. G. Andrews, "Uplink capacity and interference avoidance for two-tier femtocell networks,” IEEE Trans. Wireless Commun., vol. 8, no. 7, pp. 3498-3509, Jul. 2009. Article (CrossRef Link)

[6] V. Chandrasekhar and J. G. Andrews, "Spectrum allocation in tiered cellular networks," IEEE Trans. Commun., vol. 57, no. 10, pp. 3059-3068, Oct. 2009. Article (CrossRef Link)

[7] M. Bennis, S. M. Perlaza, P. Blasco, et al, "Self-organization in small-cell networks: A reinforcement learning approach,” IEEE Trans. Wireless Commun., vol. 12, no. 7, pp. 3202-3212, 2013. Article (CrossRef Link)

[8] C. Wang, et al, "Stackelberg game for spectrum reuse in the two-tier LTE femtocell network," in Proc. of IEEE WCNC 2013, 2013. Article (CrossRef Link)

[9] P. Semasinghe, E. Hossain and K. Zhu, “An evolutionary game for distributed resource allocation in self-organizing small-cells,” IEEE Trans. Commun., vol. 14, no. 2, pp. 274-287, 2014. Article (CrossRef Link)

[10] W. Ni, and I. B. Collings, “A new adaptive small-cell architecture,” IEEE J. Sel. Areas Commun., vol. 31, no. 5, pp. 829-839, Jun. 2013. Article (CrossRef Link)

[11] P. Kulkarni, W. H. Chin, and T. Farnham, "Radio resource management considerations for LTE Femto cells,” ACM SIGCOMM Comput. Commun., vol. 40, no. 1, pp. 26-30, Jan. 2010. Article (CrossRef Link)

[12] S. Y. Lien, C. C. Tseng, K. C. Chen, and C. W. Su, "Cognitive radio resource management for QoS guarantees in autonomous femtocell networks,” in Proc. of IEEE Int. Conf. Commun., pp. 1-6, May 2010. Article (CrossRef Link)

[13] H. C. Lee, D. C. Oh, and Y. H. Lee, "Mitigation of inter-femtocell interference with adaptive fractional frequency reuse,” in Proc. of IEEE Int. Conf. Commun., pp. 1-5, May 2010. Article (CrossRef Link)

[14] K. Sundaresan and S. Rangarajan, "Efficient resource management in OFDMA femtocells," in Proc. of ACM Int. Symp. Mobile Ad Hoc Netw. Comput., pp. 33-42, May, 2009. Article (CrossRef Link)

[15] Y. S. Liang, et al. "Resource allocation with interference avoidance in OFDMA femtocell networks,” IEEE Transactions on Vehicular Technology, vol. 61, no. 5, pp. 2243-2255, 2012. Article (CrossRef Link)

[16] A. Abdelnasser, E. Hossain, and D. I. Kim, "Clustering and resource allocation for dense femtocells in a two-tier cellular OFDMA network,” IEEE Trans. Wireless Commun., vol. 13, no. 3, pp. 1628-1641, 2014. Article (CrossRef Link)

[17] X. Kang, R. Zhang, and M. Motani, "Price-based resource allocation for spectrum-sharing femtocell networks: A stackelberg game approach,” IEEE J. Selected Areas in Commun., vol. 30, no. 3, pp. 538-549, Apr. 2012. Article (CrossRef Link)

[18] F. Pantisano, M. Bennis, W. Saad, M. Debbah,et al., "Coalition formation games for femtocell interference management: A recursive core approach,” in Proc. of IEEE WCNC, pp. 1161-1166,2011. Article (CrossRef Link)

[19] F. Pantisano, M. Bennis, W. Saad, M. Debbah,et al., "Interference alignment for cooperative femtocell networks: A game-theoretic approach,” IEEE Trans. Commun., vol. 52, no. 5, pp. 61-67, 2014. Article (CrossRef Link)

[20] P. Semasinghe, E. Hossain and K. Zhu, “An evolutionary game for distributed resource allocation in self-organizing small-cells,” IEEE Trans. Commun., vol. 14, no. 2, pp. 274-287, 2014.

[21] Q. Ni, C. Zarakovitis, "Nash Bargaining Game Theoretic Scheduling for Joint Channel and Power Allocation in Cognitive Radio Systems," IEEE Journal on Selected Areas in Communications, vol. 30, No. 1, pp. 70-81, 2012. Article (CrossRef Link)

[22] Y. H. Xu, Q. H. Wu, L. Shen, et al, “Opportunistic spectrum access with spatial reuse: Graphical game and uncoupled learning solutions,” IEEE Trans. Wireless Commun., vol. 12, no. 10, pp. 4814-4826, 2013. Article (CrossRef Link)

[23] Y. H. Xu, J. Wang, Q. H. Wu, et al, “Opportunistic spectrum access in cognitive radio networks: Global optimization using local interaction games,” IEEE Journal of Selected Topics in Signal Processing , vol. 6, No. 2, pp 180-194, 2012. Article (CrossRef Link) 
[24] J. C. Zheng, Y. M. Cai, and Y. H. Xu, "MAC-layer interference mitigation in dynamic and distributed environment: dynamic graphic game with stochastic learning," in Proc. of 2014 5th International Conference on Game Theory for Networks (GAMENETS), IEEE, pp. 1-6, 2014. Article (CrossRef Link)

[25] Y. H. Xu, Q. H. Wu, and L. Shen et al, "Robust multiuser sequential channel sensing and access in dynamic cognitive radio networks: potential games and stochastic learning," IEEE Transactions on Vehicular Technology, vol. 64, no. 8, pp. 3594 - 3607, 2015. Article (CrossRef Link)

[26] Y. H. Xu, C. G. Wang and J. H. Chen et al, "Load-aware dynamic spectrum access for small cell networks: A graphical game approach,” IEEE Transactions on Vehicular Technology, to appear. Article (CrossRef Link)

[27] C. Zarakovitis and Q. Ni, "Maximising Energy Efficiency in Multi-User Multi-Carrier Broadband Wireless Systems: Convex Relaxation and Global Optimisation Techniques," IEEE Transactions on Vehicular Technology, to appear, 2015. Article (CrossRef Link)

[28] H. Pervaiz, L. Musavian and Q.Ni, et al "Energy and Spectrum Efficient Transmission Techniques under QoS Constraints towards Green Heterogeneous Networks," IEEE Access Journal, vol. 3, pp. 1655-1671, Oct 2015. Article (CrossRef Link)

[29] S. Buzzi, G. Colavolpe, D. Saturnino, and A. Zappone, "Potential games for energy-efficient power control and subcarrier allocation in uplink multicell OFDMA systems," IEEE J. Sel. Topics Signal Process., vol. 6, no. 2, pp. 89-103, Apr. 2012. Article (CrossRef Link)

[30] H. Tembine, Distributed strategic learning for wireless engineers, CRC Press, 2012. Article (CrossRef Link)

[31] Y. Xu, J. Wang and Q. Wu, et al., "Dynamic spectrum access with statistical QoS provisioning: A distributed learning approach beyond expectation optimization," available: http://arxiv.org/abs/1502.06672. Article (CrossRef Link)

[32] R. Jain, D. Chiu, and W. Haws, "A quantitative measure of fairness and discrimination for resource allocation in shared computer system,” Technical Report, 1984. Article (CrossRef Link) 


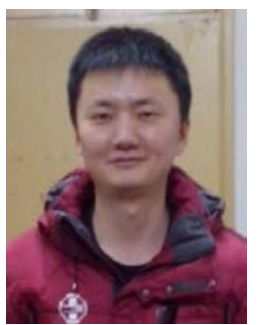

Ducheng Wu received his B.S. degree in electronic and information engineering from Wuhan University of Technology, Wuhan, China, in 2010 and M.S. degree from College of Communications Engineering, PLA University of Science and Technology, Nanjing, China, in 2013, respectively. He is currently a Ph.D. candidate in College of Communications Engineering, PLA University of Science and Technology. His research interests include resource allocation in small cell networks, game theory and statistical learning.

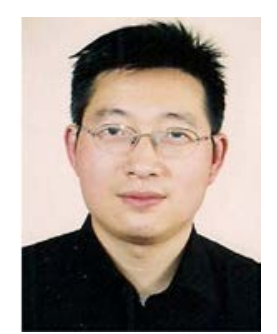

Qihui Wu received his B.S. degree in communications engineering, M.S. degree and $\mathrm{Ph} . \mathrm{D}$. degree in communications and information system from Institute of Communications Engineering, Nanjing, China, in 1994, 1997 and 2000, respectively. He is currently a professor at the PLA University of Science and Technology, China. His current research interests are algorithms and optimization for cognitive wireless networks, soft-defined radio and wireless communication systems.

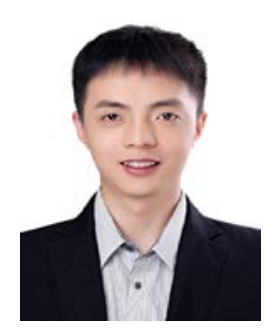

Yuhua Xu received his B.S. degree in Communications Engineering, and Ph.D. degree in Communications and Information Systems from College of Communications Engineering, PLA University of Science and Technology, in 2006 and 2014 respectively. He has been with College of Communications Engineering, PLA University of Science and Technology since 2012, and currently as an Assistant Professor. His research interests focus on opportunistic spectrum access, learning theory, game theory, and distributed optimization techniques for wireless communications. He has published several papers in international conferences and reputed journals in his research area. He is an Editor for the KSII Transactions on Internet and Information Systems. In 2011 and 2012, he was awarded Certificate of Appreciation as Exemplary Reviewer for the IEEE Communications Letters. 